\title{
QOS PERFORMANCE OF WR-OBS NETWORK ARCHITECTURE WITH REQUEST SCHEDULING
}

\author{
Eugene Kozlovski and Polina Bayvel \\ Optical Network Group, Department of Electronic and Electrical Engineering, University \\ College London, Torrington Place, London WC1E 7JE, UK \\ \{ekozlovs, pbayvel\} @ee.ucl.ac.uk
}

\begin{abstract}
The paper investigates the QoS performance with service differentiation of the wavelength-routed optical burst switched (WR-OBS) network architecture. The request scheduler was analysed as the means of the QoS provisioning and impact of the request scheduling on the QoS performance was investigated in terms of the burst blocking probability and burst end-to-end delays. The WROBS QoS performance was also studied as a function of the wavelength requirement. The trade-off between the wavelength over-provisioning and the maximum scheduling delay satisfying the service requirements is reported for both premium and best-effort traffic classes. It is shown that the request scheduling allows to significantly improve the QoS performance and reduce the wavelength requirement in the WR-OBS.
\end{abstract}

Key words: Optical Burst Switching, Quality of Service, Service Differentiation, Dynamic Wavelength Routing and Assignment, Wavelength Requirement

\section{INTRODUCTION}

The optical burst-switching (OBS) approach for the IP-over-WDM network architecture has been extensively investigated due to its ability to provide potentially better utilisation of optical bandwidth compared with statically routed WDM networks [1-3]. With the OBS, packets are buffered in the electronic domain and then the aggregated bursts of packets are transmitted over the WDM optical core. However, most currently proposed OBS schemes, such as the JET [4], suffer from high burst blocking rate for high traffic loads and assume full wavelength conversion [5]. Moreover, 
whilst the OBS has been demonstrated to allow for the QoS provisioning, it has been shown that an improvement of the burst blocking probability for high-priority traffic can only be achieved by trading off the blocking probability of low-priority traffic [6], thus degrading the performance of the whole network.

To overcome these limitations and provide the QoS-guaranteed and differentiated services, the wavelength-routed optical burst-switched architecture (WR-OBS) was proposed [7]. WR-OBS is designed to take advantage of the dynamic wavelength routing and assignment (DRWA) which allows to improve bandwidth utilisation whilst avoiding the burst processing in the optical domain.

Previous research of the WR-OBS architecture was focused on the packet loss rate in case of the finite buffers in the edge-routers [7], the bandwidth utilisation and the wavelength re-use [8] and the methods of the burst aggregation [9]. The impact of the DRWA calculation time on those methods has been reported [10]. An architecture of the request server residing in the control node was introduced in [11] in order to carry out the WR-OBS QoS provisioning. However, the provision of the guaranteed services with QoS differentiation in the WR-OBS has not yet been addressed.

In this paper, we evaluate the QoS performance in terms of both the burst blocking probability and the burst end-to-end delay for a centralised WROBS architecture with burst scheduling. We investigate the impact of the request scheduling delay (which is one of the WR-OBS controllable parameters) on the QoS provisioning. We then analyse the trade-off between the maximum scheduling delay and the burst blocking probability with service differentiation between high priority and low priority traffic classes of service. We demonstrate that the request scheduling residing in the WROBS control node allows to achieve a highly efficient QoS performance for both traffic classes in terms of the burst blocking probability whilst keeping end-to-end delays below the bounds allowed in packet-switched networks. We believe this gives the WR-OBS an advantage compared to the other OBS schemes, as the latter imply a high burst blocking probability rate for the best-effort traffic which results in higher traffic loads and higher overheads in the TCP/IP or other transmission protocols due to the required re-transmission of blocked bursts.

Additionally, a comparison between the performance of the dynamic wavelength assignment (fast circuit switching) and quasi-static wavelength assignment was analysed in terms of the wavelength requirement in the core network. The relationship between the WR-OBS QoS performance and the wavelength requirement is reported. 


\section{THE WR-OBS MODEL}

The modelled network consists of $N$ edge-routers located at the ingress of the optical core network (Fig. 1). It is assumed that each edge-router is connected to an optical core router. One of the edge-routers also carries out the function of the control node, or request server, responsible for wavelength assignment to requests arriving from edge-routers [12].

Each request signalled to the request server represents a burst of IP packets aggregated in the edge-router and directed to the same destination. Each edge-router has $C(N-1)$ electronic buffers where $C$ is the number of traffic classes of service (CoS). One buffer per destination per $\operatorname{CoS}$ is assumed, where packets coming from the access layer are aggregated to bursts. As soon as the burst has been aggregated, the request for a lightpath is sent to the request server.

Several options for the burst aggregation mechanism have been proposed $[9,10]$. In this paper we consider a variation of Fixed Burst Aggregation Time (FBAT) method. In the FBAT, burst size is limited by a time-out signal which is issued once the defined fixed time of burst aggregation, aggregation delay $t_{\text {aggr, }}$ has been exceeded. Thus, the time-out signal interrupting the aggregation is issued at equal $t_{\text {aggr }}$ periods of time. Shortly before $t_{\text {aggr }}$ elapses, a request is initiated and sent to the request server with an estimate information of the burst size. Once the time-out signal has been generated, a new cycle starts for the aggregation of the next burst. The transmission of the burst begins as soon as the edge node receives an acknowledgement signal from the request server notifying that a lightpath for the transmission has been established. It should be noted that the FBAT allows the request server to take into account an estimate of the burst size. Moreover, the precise burst size can be delivered to the request server once the time-out signal has been issued. As this information can be used to calculate the time required for lightpath allocation, the request server can carry out the resource scheduling in the most efficient way. However, it can be shown that using the FBAT imposes higher requirements on the DRWA calculation time with regards to other burst aggregation schemes.

In the request server, requests are first sorted according to their $\operatorname{CoS}$ so that requests of a given $\mathrm{CoS}$ are directed to a queue dedicated to that $\mathrm{CoS}$ (Fig. 2). QoS provisioning in the request server is based on two main functions:

- A scheduling discipline for the request sequencing inside each queue.

The modified earliest deadline first (MEDF) discipline [11] is applied as explained in section 4 . 
- A fair queueing algorithm carrying out the queues sequencing. The weighted round-robin (WRR) algorithm [13] is applied for this purpose (see section 5).

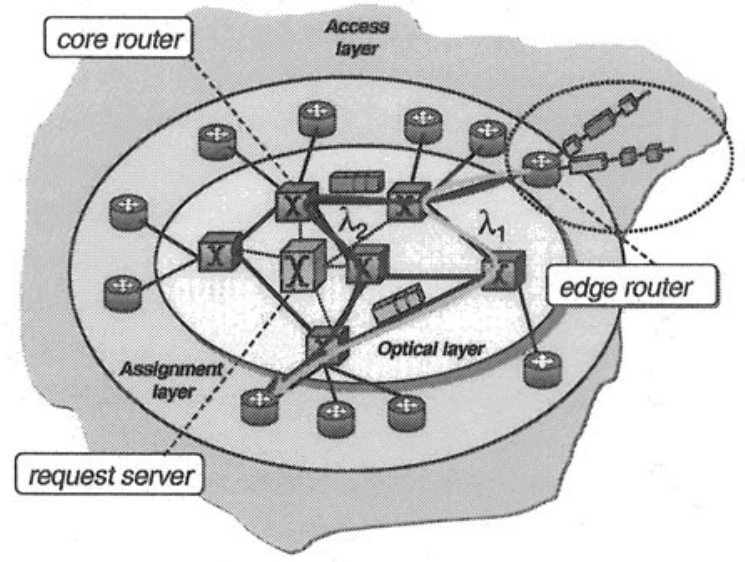

Figure 1. WR-OBS architecture

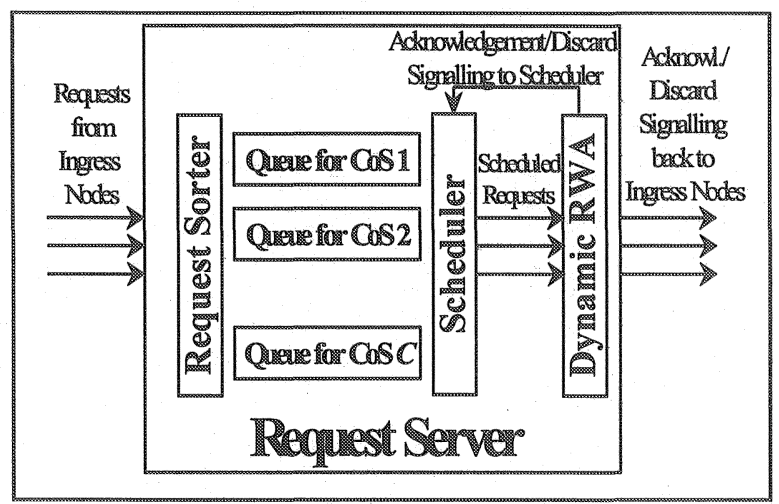

Figure 2. Request Server Architecture

While the first function provides fairness in the burst servicing within one $\mathrm{CoS}$, the second one carries out actual service differentiation between different traffic classes. The scheduled requests are then directed to the DRWA component which carries out the lightpath allocation.

We define the burst end-to-end delay, $T_{\text {EEdelay, }}$ as the time elapsed from the start of the burst aggregation until it is delivered to the destination. As shown below, this parameter depends on the burst edge delay, $T_{\text {edge, }}$ defined 
as the time elapsed from when the first packet has arrived to the buffer until the start of its transmission. delay:

In addition to $t_{\mathrm{aggr}}$, the following parameters define the burst end-to-end

- Acknowledgment time, $t_{\text {ack, }}$ is imposed by the propagation delay. It is the time elapsed from when a lightpath has been allocated by the request server until the acknowledgement signal is received by the edge-router.

- Calculation time, $t_{\text {calc, }}$ is the time required by the request server to complete the DRWA algorithm allocating a lightpath to a request.

- Transmission dealy, $t_{\text {trans }}\left(C_{i}\right)$, is the time of the transmission of the burst of $C_{i}$-class to its destination.

- Maximum request scheduling delay, $t_{\text {sched,max }}\left(C_{i}\right)$ is the maximum time allowed for the request of the traffic class $C_{i}$ to be buffered at the queue in the request server [11]. If $t_{\text {sched,max }}$ is exceeded, the request is discarded and a burst corresponding to this request is blocked. As demonstrated below, $t_{\text {sched,max }}$, is one of the most critical parameters effecting the WROBS QoS performance as well as the efficiency of the DRWA.

It should be mentioned that the access layer delays are assumed to be negligible. This way, in the case of the FBAT method, the maximum edge delay of the bursts of the traffic class $C_{i}$ is

$$
\mathrm{T}_{\text {edge,max }}\left(\mathrm{C}_{\mathrm{i}}\right)=\mathrm{t}_{\text {aggr }}\left(\mathrm{C}_{\mathrm{i}}\right)+\mathrm{t}_{\text {sched,max }}\left(\mathrm{C}_{\mathrm{i}}\right)+\mathrm{t}_{\text {calc }}+\mathrm{t}_{\text {ack, }}
$$

and the maximum end-to-end delay of the bursts of the traffic class $C_{i}$ is

$$
T_{\text {EEdelay,max }}\left(C_{i}\right)=T_{\text {edge,max }}\left(C_{i}\right)+t_{\text {trans }}\left(C_{i}\right)+t_{\text {prop, }}
$$

where $t_{\text {prop }}$ is the propagation time from a source to a destination.

Whilst assuming that $t_{\text {calc, }} t_{\text {prop }}$ and $t_{\text {ack }}$ are fixed parameters of a given network topology, in this work we optimise $t_{\text {sched,max }}\left(C_{i}\right)$ in order to provide the desired performance of the DRWA for each traffic class. We investigated the impact of this parameter on the QoS provisioning in WR-OBS in terms of the end-to-end delays and burst blocking probability (BBP). In particular, we evaluated the performance of described network architecture by means of simulation. A discrete-event WR-OBS simulator was developed in $\mathrm{C}++$. This allowed to analyse the aggregation delay as a function of the BBP as well as to investigate the relationship between the maximum request scheduling delay and the BBP for multiple classes of service and in terms of the core wavelength provisioning, $W_{\text {dynamic }}$ (wavelength requirement for the DRWA, introduced in section 3). The reported results were obtained with the confidence of $95 \%$. 
The First-Fit heuristic was used for the dynamic RWA component. This heuristic combines low computational complexity with low request blocking rate [14].

The ARPA Net physical topology with $N=20$ nodes was used to investigate the WR-OBS QoS performance on a realistic network. The bursts of each traffic class were aggregated out of packets generated with the $\mathrm{ON}$ OFF Pareto model as in [7]. It was assumed that

$$
D_{\mathrm{ON}}(U)=\left\lfloor\frac{X_{0}}{U^{1 / \alpha}}\right\rfloor, \quad D_{\mathrm{OFF}}(U)=\left\lfloor\frac{X_{0}}{U^{1 / \alpha}} \cdot \frac{1-\rho}{\rho}\right\rfloor,
$$

where $\rho$ is the input traffic load, $D_{\mathrm{ON}}$ is the packet size in bits, $D_{\text {OFF }}$ is the number of void bits representing the inter-arrival time, $U$ is a random variable uniformly distributed between 0 and $1, \alpha=1.5$ and $X_{0}=1333\left(X_{0}\right.$ represents minimum packet size in bits). This way, the average packet size is about 500 bytes [15].

We consider the case of infinite-size buffers for the burst aggregation. In this way, the burst size is only limited by the aggregation delay $t_{\text {aggr }}\left(C_{i}\right)$ which is set to $40 \mathrm{~ms}$ for each traffic class throughout the paper. The average burst size in our simulations is in the range of tens of milliseconds for the burst transmission through the network.

Uniform distribution of traffic loads between the edge-routers was assumed. We also assumed zero calculation time of the RWA algorithm, i.e. $t_{\text {calc }}=0$ throughout the paper. Additionally, the propagation delay $t_{\text {prop }}$ and the acknowledgement time $t_{\text {ack }}$ are assumed to be $2.5 \mathrm{~ms}$ for all the links.

\section{DRWA EFFICIENCY}

In this work, we defined the wavelength requirement in the dynamicallyrouted WDM networks, $W_{\text {dynamic}}$, as the number of wavelengths in the core required to achieve a given burst blocking probability. The parameter $W_{\text {dynamic }}$ imposes a constraint on the QoS performance because the bandwidth available to accommodate the bursts is limited by the number of the core wavelengths. The efficiency of the DRWA in terms of $W_{\text {dynamic }}$ can be compared with the statically-routed network architecture the folowing way.

Let $W_{\text {static }}$ be the wavelength requirement for an architecture based on the static routing and wavelength assignment (SRWA). In the SRWA, the wavelengths are permanently (or quasi-statically) assigned to the lightpaths 
for each node-pair as in [16]. Then $W_{\text {static }}$ is an upper bound on the wavelength provisioning in the core required for the loss-free and delay-free burst transport. Let also $B_{\text {in,max }}$ be the maximum bit-rate of the total traffic arriving at one node and let $b_{\text {in,max }}$ be the maximum bit-rate of the traffic arriving at one buffer. Then the following applies:

$$
B_{\text {in, } \max }=C \cdot(N-1) \cdot b_{\text {in, } \max }
$$

We define $W\left(B_{\mathrm{in}, \max }\right)$ as the minimum number of the wavelengths in the core required to accommodate all the traffic arriving from one edge router in the network with a given physical topology if this traffic has to be transmitted over the same optical link. This represents the worst case of the lightpath allocation. The parameter $W\left(B_{\mathrm{in}, \max }\right)$ can be given as:

$$
\mathrm{W}\left(\mathrm{B}_{\mathrm{in}, \max }\right)=\left\lceil\frac{B_{\text {in,max }}}{b_{\text {core }}}\right\rceil=\left\lceil\frac{C \cdot(N-1) \cdot b_{\text {in, } \max }}{b_{\text {core }}}\right\rceil,
$$

where $b_{\text {core }}$ is the bandwidth used by one core wavelength. Then the following condition holds:

$$
W\left(B_{\text {in,max }}\right) \leq W_{\text {dynamic }} \leq W_{\text {static. }}
$$

This condition implies that the bandwidth occupied by the total input traffic should not exceed the bandwidth of the core optical link. If the number of the wavelengths in the core equals $W\left(B_{\text {in,max }}\right)$, the network is said to have no wavelength over-provisioning. On the other hand, $W_{\text {dynamic }}$ should be lower than $W_{\text {static, }}$ otherwise the dynamic RWA leads to poorer bandwidth utilisation and brings no advantage compared to the static RWA.

Let $P_{\mathrm{b}}\left(C_{i}\right)$ be the maximum $\mathrm{BBP}$ imposed by the requirement of the service $C_{i}$. This way, if $P_{\mathrm{b}}\left(C_{i}\right)$ is achieved by the WR-OBS, the efficiency of the DRWA with regards to the static assignment is: 


$$
E_{\mathrm{DA}}=\frac{W_{\text {static }}-W_{\text {dynamic }}}{W_{\text {static }}-W\left(B_{\text {in, } \max }\right)} \cdot 100 \%
$$

Eq. (7) allows to define the wavelength over-provisioning as a wavelength requirement with $W_{\text {dynamic }}>W\left(B_{\text {in,max }}\right)$, i.e with $E_{\mathrm{DA}}<100 \%$ 。

\section{IMPACT OF THE SCHEDULING DELAY ON THE EFFICIENCY OF THE DRWA ALGORITHM}

In this section, we analyse the request scheduling at the request server in order to optimise the performance of the DRWA algorithm with respect to the static assignment whilst taking into account the WR-OBS QoS provisioning (in terms of the BBP and burst end-to-end delays).

The parameter $E_{\mathrm{DA}}$ (eq. (7)) allows to investigate how the request scheduling influences the wavelength utilisation in the WR-OBS. In our analysis, the request scheduling for each queue in the request server is based on the modified earliest-deadline-first (MEDF) discipline [11]. Once a lightpath has been released, this discipline applies the following procedures to each request in the queues:

1. If the request of a given traffic class $C_{i}$ has exceeded maximum scheduling delay, $t_{\text {sched,max }}\left(C_{i}\right)$, the server drops it and sends a discard signal back to the edge-router.

2. Otherwise, it attempts to allocate a lightpath and, if successful, it sends acknowledgement to the edge-router. The latter then sends the burst over the assigned lightpath through the core network without intermediate optical processing.

3. Once the entire burst has been transmitted, the lightpath is released.

4. If the request is blocked by RWA but it has not exceeded $t_{\text {sched,max }}\left(C_{i}\right)$, it is sent back to the queue and when the next lightpath is released, the queue is re-ordered so that a request of the earlier-aggregated burst is served prior to that of the later-aggregated burst. This way, the burst fair queuing is achieved.

Throughout this analysis the traffic was generated using eq. (3) with $b_{\text {in, } \max }=b_{\text {core }}=10 \mathrm{Gbit} / \mathrm{s}$. The value of the $\mathrm{BBP}=10^{-4}$ is chosen to limit the simulation computational time, i.e. $P_{\mathrm{b}}=10^{-4}$. However, the relationships demonstrated below can also be achieved with lower $P_{\mathrm{b}}$ values. Again, in this section only one class of service is considered because the demonstrated 
principle applies to any CoS. Thus, $C=1, W\left(B_{\text {in,max }}\right)=19$ (see eq. (5)) and $W_{\text {static }}=33$ for the ARPA Net physical topology [16].

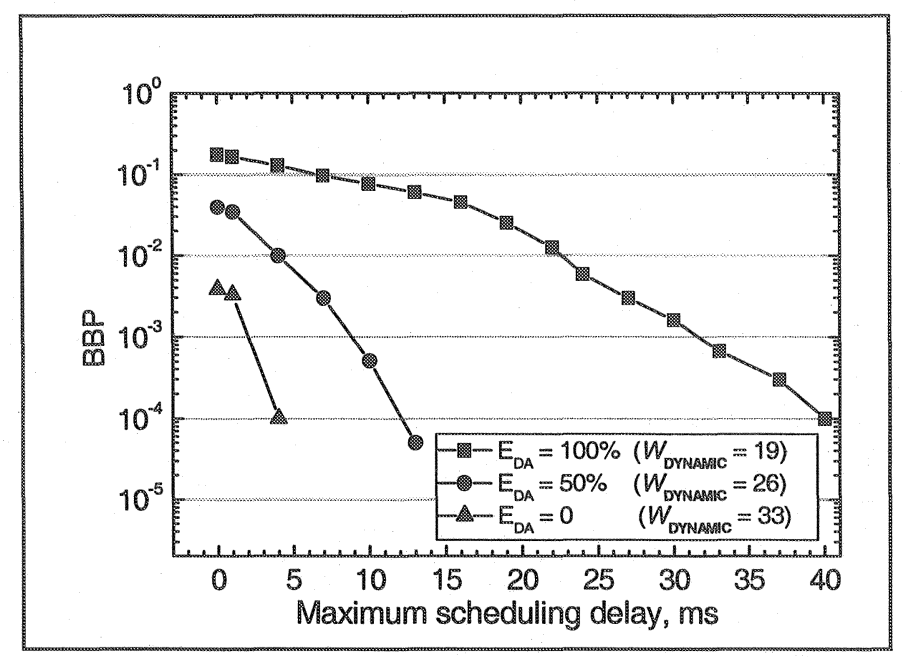

Figure 3. The BBP vs. $t_{\text {sched,max }}$ for different $E_{\mathrm{DA}}$, aggregation delay $t_{\mathrm{aggr}}=40 \mathrm{~ms}$, Traffic $\operatorname{Load}=0.7$.

The relationship between the BBP and the $t_{\text {sched,max }}$ for different values of $E_{D A}$ is shown in Fig. 3. It can be seen that the decrease in $t_{\text {sched,max }}$ significantly adds to the blocking probability. It can be observed that when $t_{\text {sched,max }}=0$, the BBP of $10^{-4}$ is not reached even for $E_{D A}=0$ (i.e. when $W_{\text {dynamic }}=W_{\text {static }}$ ). This indicates that the dynamic wavelength assignment has very limited capabilities when not supported by the request scheduling in the central node. In contrast, if $t_{\text {sched,max }}$ of $40 \mathrm{~ms}$ is allowed (which represents the case of $t_{\text {sched,max }}=t_{\text {aggr }}$ ), the $\mathrm{BBP}=P_{\mathrm{b}}$ for $E_{D A}=100 \%$, i.e. in this case the DRWA outperforms the static assignment, because no wavelength overprovisioning is required in this case at all.

Fig. 4 shows how the core wavelength over-provisioning influences the BBP under the different values of $t_{\text {sched,max }}$. Again, it can be seen that with $t_{\text {sched,max }}=0$, no over-provisioning for $E_{D A}>0$ can achieve the desired value of the BBP, whilst with $t_{\text {sched,max }}=20 \mathrm{~ms}$, the BBP $=P_{\mathrm{b}}$ is achieved for 24 wavelengths, i.e. for $E_{D A}>50 \%$. It should be noticed that further increase in $t_{\text {sched,max }}$ will lead to significant end-to-end delays (exceeding $100 \mathrm{~ms}$ ) and would not be acceptable for QoS-sensitive traffic. 


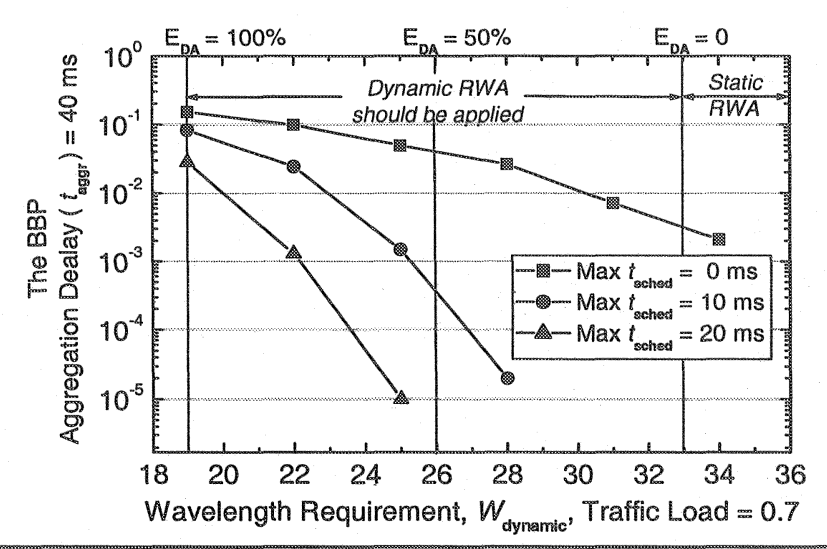

Figure 4. The BBP vs. the wavelength requirement for different $t_{\text {sched,max }}$

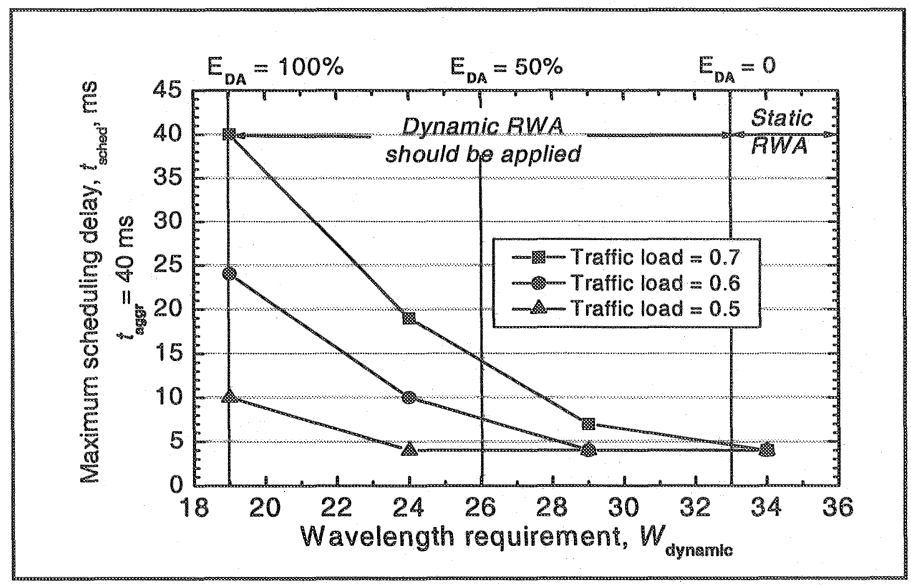

Figure 5. The trade-off between $t_{\text {sched,max }}$ and the wavelength requirement for different traffic loads, the $\mathrm{BBP}<10^{-4}$ for all curves

The relationship between $t_{\text {sched,max }}$ and $W_{\text {dynamic }}$ with the $\mathrm{BBP} \leq P_{\mathrm{b}}$ is shown in Fig. 5 which demonstrates the trade-off between the core wavelength over-provisioning and QoS in terms of end-to-end delay. Based on this graph, optimal values of $W_{\text {dynamic }}$ and $t_{\text {sched,max }}$ can be chosen depending on the particular QoS requirements. The relationship shows that $t_{\text {sched,max }}$ as small as $14 \mathrm{~ms}$ decreases the wavelength requirement twice, i.e. $E_{D A}$ of $50 \%$ is reached. 
The above results show that the DRWA should be used only in combination with the request scheduling in the control node. This way, it significantly outperforms static RWA in terms of the wavelength requirement whilst reaching the negligible blocking probability and small end-to-end delays.

\section{THE QOS PROVISIONING IN WR-OBS WITH SERVICE DIFIERENTIATION}

We now investigate the service differentiation in WR-OBS between the premium and the best effort classes of traffic. In this analysis packets of both classes were generated with equal probability. Thus, the amount of premium traffic is $50 \%$ of the total traffic. This value is selected to demonstrate that even if the proportion of the premium traffic is high, it can be effectively provided with a guaranteed service.

As previously mentioned, the weighted round robin (WRR) algorithm was implemented in the request server in order to carry out the service differentiation by means of the queues sequencing. In the WRR, the premium traffic queue is given the weight of $90 \%$ and the best-effort traffic queue is given the weight of $10 \%$. This way, once a lightpath has been released, the queues are ordered so that each best-effort traffic request is served only when nine premium traffic requests have been served.

For the ARPA-Net physical topology, when $C=2$ each edge-router can establish at most 38 lightpaths simultaneously, i.e. $W\left(B_{\text {in, max }}\right)=38$ (see eq. (5)). For the case of two classes, two lightpaths can be established simultaneously between each node-pair. Therefore we assume that the total input traffic is doubled with respect to that of one CoS. This way, in the case of two classes, $W_{\text {static }}=66$. Let $C_{1}$ denote the premium class and $C_{2}$ denote the best-effort class. As in Section 4, here it is assumed that $P_{\mathrm{b}}\left(C_{1}\right)=P_{\mathrm{b}}\left(C_{2}\right)$ $=10^{-4}$ and that $t_{\text {aggr }}\left(C_{1}\right)=t_{\text {aggr }}\left(C_{2}\right)=40 \mathrm{~ms}$. Uniform traffic loads of 0.7 are considered in this section.

It can be said that WR-OBS performs efficiently in terms of the wavelength requirements or resource utilisation if $E_{\mathrm{DA}}$ greater than $50 \%$ can be achieved. Otherwise, a quasi-static wavelength routing should be used. Thus, wavelength over-provisioning with lower values of $E_{\mathrm{DA}}$ than $50 \%$ is not considered in this section.

The maximum scheduling delay $t_{\text {sched,max }}\left(C_{i}\right)$ should be chosen from the service requirement on the $C_{i}$ end-to-end delay and it varies for each $\operatorname{CoS}$. The MEDF discipline (explained in Section 4) thus operates with variable values of $t_{\text {sched,max }}$ for each $C_{i}$. The case of $t_{\text {sched, } \max }\left(C_{1}\right)=t_{\text {sched,max }}\left(C_{2}\right)=10 \mathrm{~ms}$ demonstrating the WRR impact on the service differentiation is shown in 
Fig. 6 as a function of $W_{\text {dynamic. }}$. It can be seen that the difference in the BBP of more than two orders of magnitude between the two classes is achieved by the WRR scheduling in the server. However, the BBP for the best-effort traffic remains very high even for $E_{\mathrm{DA}}=50 \%$, i.e. even with a significant wavelength over-provisioning.

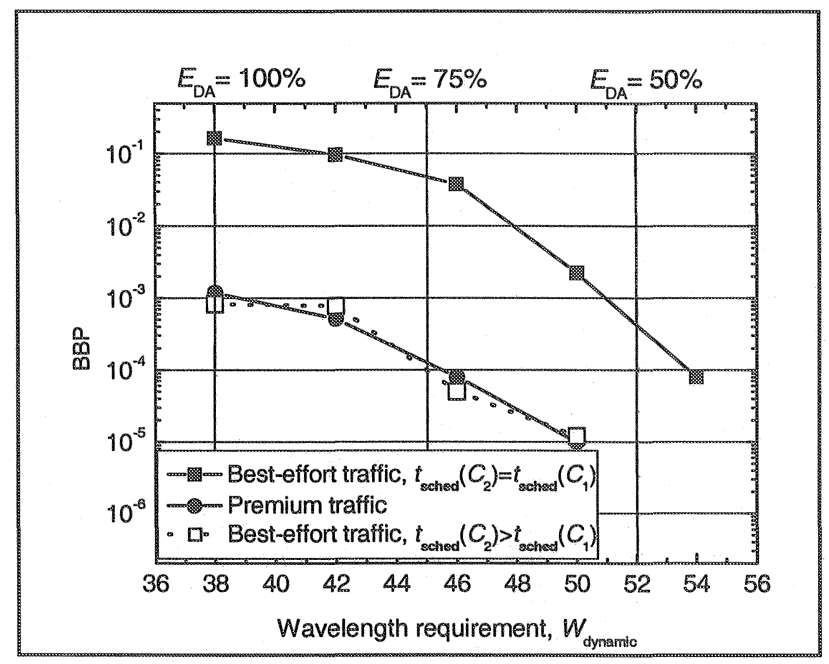

Figure 6. The BBP vs. $W_{\text {dynamic }}$ for both $\operatorname{CoS}$

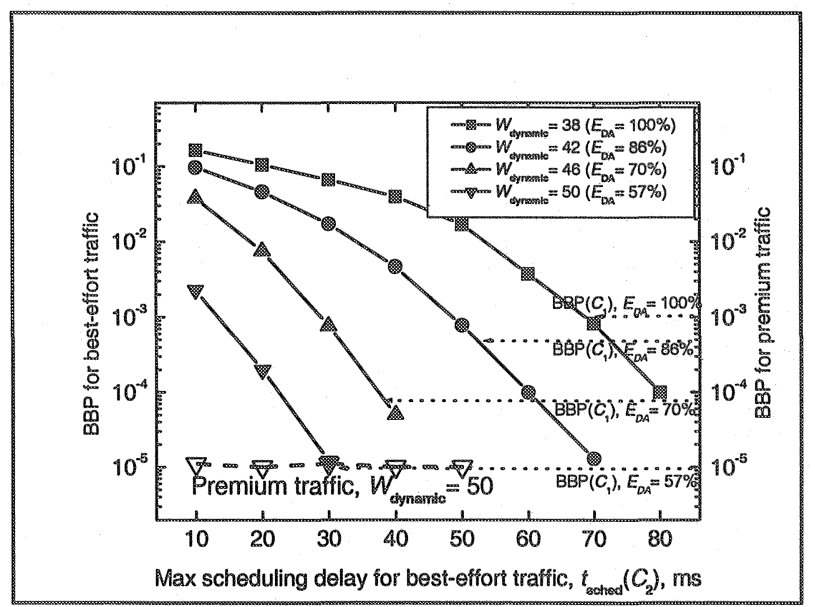

Figure 7. Best-effort traffic BBP vs. $t_{\text {sched }}\left(C_{2}\right), t_{\text {sched }}\left(C_{1}\right)=10 \mathrm{~ms}$. Dashed line denotes the $\mathrm{BBP}\left(C_{1}\right)\left(\mathrm{BBP}\right.$ of premium traffic) with $W_{\text {dynamic }}=50$. Dotted lines represent the $\operatorname{BBP}\left(C_{1}\right)$ for different $W_{\text {dynamic }}$ and allow to determine appropriate values of $t_{\text {sched }}\left(C_{2}\right)$ reaching the BBP $\left(C_{2}\right)=\mathrm{BBP}\left(C_{1}\right)$ 
Nevertheless, the desired BBP of the best-effort traffic can be achieved even for $E_{\mathrm{DA}}=100 \%$ by increasing $t_{\text {sched,max }}\left(C_{2}\right)$. This approach is demonstrated in Fig. 7 which shows the relationship between the BBP for the best-effort traffic and $t_{\text {sched,max }}\left(C_{2}\right)$ for different values of $W_{\text {dynamic }}$. As the end-to-end delay of the time-sensitive premium traffic cannot be increased, the value of $t_{\text {sched,max }}\left(C_{1}\right)$ remains $10 \mathrm{~ms}$ and is constant for all curves. The dotted lines in Fig. 7 allow to determine the values of $t_{\text {sched,max }}\left(C_{2}\right)$ required for the best-effort traffic to achieve approximately the same BBP as that of the premium traffic shown in Fig. 6. The BBP of the best-effort traffic with the increased $t_{\text {sched,max }}\left(C_{2}\right)$, approximating the BBP of the premium traffic, is plotted in Fig. 6 by the dotted line. Thus, the request scheduling allows for both traffic classes to keep the same BBP at the expense of the increased end-to-end delay of the best-effort traffic. It can be seen that even in the case of $E_{\mathrm{DA}}=100 \%$, the $\mathrm{BBP}=P_{\mathrm{b}}\left(C_{2}\right)$ is achieved for the best-effort traffic when $t_{\text {sched,max }}\left(C_{2}\right)=80 \mathrm{~ms}=2 t_{\text {aggr }}$, which is still acceptable for the non-real-time applications [17]. It can also be shown that under the described configuration of the request scheduler, the increase in $t_{\text {sched,max }}\left(C_{2}\right)$ does not affect the QoS of the premium traffic. Fig. 7 demonstrates that whilst the BBP of the besteffort traffic is reduced by the increase of $t_{\text {sched,max }}\left(C_{2}\right)$, for any values of $t_{\text {sched,max }}\left(C_{2}\right)$ the BBP of the premium traffic remains constant (plotted by the dashed line). Thus, the WR-OBS request server can be configured so that the QoS provisioning in terms of the BBP is carried out independently for each CoS when guaranteed service differentiation between two traffic classes is required.

Based on the given results, a trade-off can be obtained between the maximum scheduling delay and the core wavelength over-provisioning allowing the BBP $<P_{\mathrm{b}}\left(C_{i}\right)$ for both traffic classes at a time, as shown in Fig.8. On this diagram, the value of $W_{\text {dynamic }}$ required to achieve the desired BBP can be determined for different values of $t_{\text {sched,max }}\left(C_{1}\right)$ and $t_{\text {sched,max }}\left(C_{2}\right)$ allowed by the QoS requirements. As can be seen from Fig. 8, for $t_{\text {sched,max }}\left(C_{1}\right)=20 \mathrm{~ms}$ and $t_{\text {sched,max }}\left(C_{2}\right)=80 \mathrm{~ms}$, the desired BBP can be achieved with no wavelength over-provisioning at all, i.e. with $W_{\text {dynamic }}=$ $W\left(B_{\text {in,max }}\right)$. If, however, lower values of $t_{\text {sched, } \max }\left(C_{1}\right)$ and $t_{\text {sched,max }}\left(C_{2}\right)$ are required by a certain service, slight wavelength over-provisioning with $E_{\mathrm{DA}}=$ $75 \%$ reduces both $t_{\text {sched,max }}\left(C_{1}\right)$ and $t_{\text {sched,max }}\left(C_{2}\right)$ by as much as $50 \%$.

It should be noted that the relationship between the BBP and $t_{\text {sched,max }}$ can be improved by applying more sophisticated scheduling algorithms and the DRWA heuristics. Thus, our model is not optimal in terms of the QoS performance. However, as demonstrated above, even in this case the request scheduling with a fairly small maximum scheduling delay significantly 
reduces the wavelength requirement satisfying the desired burst blocking probability for both types of traffic.

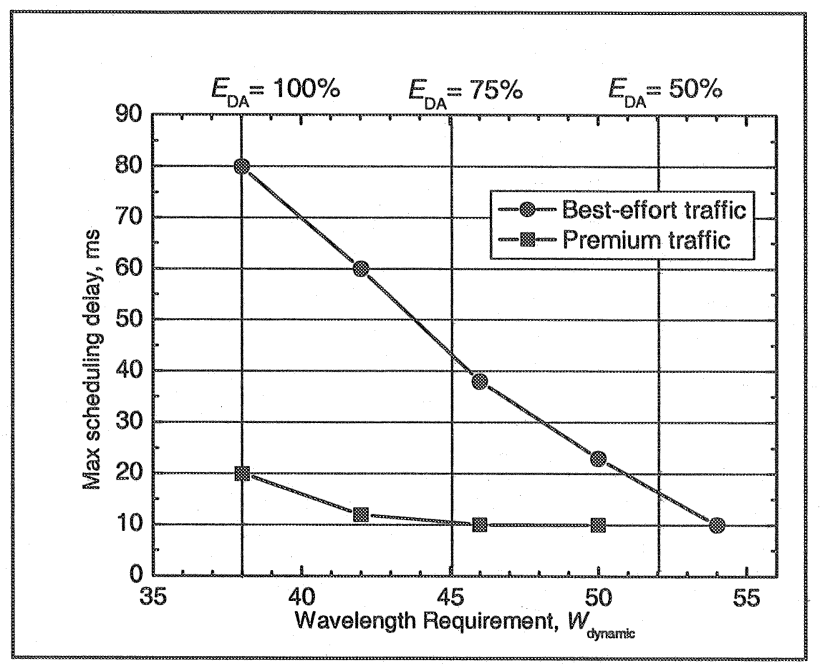

Figure 8. The trade-off between $t_{\text {sched }}$ and $W_{\text {dynamic. }}$ The BBP $<10^{-4}$ for both traffic classes.

\section{SUMMARY}

The QoS performance of the WR-OBS architecture with service differentiation was analysed in terms of the burst end-to-end delay and the burst blocking probability. The impact of the request scheduling delay on the QoS performance was investigated. It was demonstrated that the WR-OBS QoS provisioning for the premium traffic does not trade off the blocking probability of the best-effort traffic. It was shown that the implementation of the correct scheduling mechanism as part of the request server guarantees the desired blocking probability for both premium and the best-effort traffic whilst ensuring that the delay bounds for both classes are not exceeded. This indicated the operating conditions under which the WR-OBS becomes a viable network architecture, although its limitations and advantages with respect to the JET scheme are the subjects of further investigation [18] and will be described in detail elsewhere.

Additionally, the DRWA efficiency compared to the statically-routed networks was formulated and analysed in terms of the wavelength requirement. The trade-off between the wavelength over-provisioning and the maximum scheduling delays satisfying the QoS requirements was quantified. It was demonstrated that the desired QoS performance can be 
achieved for each class even with no core wavelength over-provisioning providing the small scheduling delays comparable with the aggregation delay are acceptable for the service requirement on the burst end-to-end delay.

Whilst it can be shown that the WR-OBS architecture scales well for the near-future optical backbones with up to 100 nodes, the WR-OBS scalability for higher number of nodes requires further analysis.

\section{ACKNOWLEDGMENTS}

The authors would like to express their gratitude to Prof. J. Crowcroft, Dr. R. J. Gibbens (Cambridge), Dr. M. Searle (INSIG Ltd.), Dr. R. I. Killey, M. Düser, A. Zapata (UCL), and I. De Miguel (UCL and University of Valladolid, Spain) for their invaluable comments. Financial support from the Royal Society, UCL Graduate School, Ian Karten Charitable Trust, ORS and the EU IST WINMAN Project is gratefully acknowledged.

\section{REFERENCES}

[1] C. Qiao, M. Yoo, "Choices, features and issues in optical burst switching," Optical Networks Mag., Apr.2000,pp.36-44

[2] M. Yoo, C. Qiao, S. Dixit, "QoS performance of optical burst switching in IP-over-WDM networks," IEEE Journal on Selected Areas in Communications, Vol. 18, No. 10, Oct. 2000 , pp. 2062-2071 [3] J.S. Turner, "Terabit burst switching," Journal of High-Speed Networks, Vol. 8, 1999, pp. 3-16

[3] C. Qiao, M. Yoo, "Optical bust switching (OBS) - A new paradigm for an optical Internet," Journal of High-Speed Networks, Vol. 8, 1999, pp. 69-84

[4] M. Yoo, C. Qiao, "A novel switching paradigm for buffer-less WDM networks", Proc. OFC1999, 1999, paper ThM6-1/177

[5] K. Dolzer, C. Gauger, J. Späth, S. Bodamer, "Evaluation of Reservation Mechanisms for Optical Burst Switching", AEÜ Int. J. Electron. Commun. 55 (1), 2001, 1-8

[6] K. Dolzer, C. Gauger, "On burst assembly in optical burst switched networks - a performance evaluation of Just-Enough-Time", Proc. $17^{\text {th }}$ Int. Teletraffic Congress, Sep. 2001

[7] M. Düser, E. Kozlovski, R. I. Killey, P. Bayvel, "Design trade-offs in optical burst switched networks with dynamic wavelength allocation,", Proc. ECOC 2000, pp.23-24

[8] M. Düser, P. Bayvel, "Bandwidth utilisation and wavelength re-use in WDM Optical Burst-Switched Packet Networks," Proc. ONDM 2001, Vol. 1, 2001

[9] I. de Miguel, M. Düser, P. Bayvel, "Traffic load bounds for optical burst-switched networks with dynamic wavelength allocation," Proc. ONDM 2001, Vol. 1, 2001

[10] I. de Miguel, M. Düser, P. Bayvel, "The impact of dynamic wavelength assignment and burst aggregation in optical burst-switched networks," Proc. LCS 2001, Sep. 2001, pp. $167-170$ 
[11] E. Kozlovski, M. Düser, I. de Miguel, P. Bayvel, "Analysis of burst scheduling for dynamic wavelength assignment in optical burst-switched networks," Proc. IEEE LEOS 2001 Annual Meeting, paper TuD2

[12] M. Düser, E. Kozlovski, R. Killey P. Bayvel, "Distributed router architecture for packetrouted optical networks", Proc. ONDM 2000, Feb. 2000, pp. 202-221

[13] M. Katevenis, S. Sidiropoulos and C. Courcoubetis, "Weighted Round-Robin Cell Multiplexing in a General-Purpose ATM Switch Chip", IEEE JSAC, Vol. 9, No 8 , pp 1265-1279, Octubre 1991.

[14] H. Zang, P. Jue, B. Mukherjee, "A Review of Routing and Wavelength Assignment approaches for Wavelength-Routed Optical WDM Networks," Optical Networks Mag., January $2000, \mathrm{pp} .47-60$

[15] Y. Xiong, M. Vandenhoute, H.D. Cankaya, "Control architecture in optical burstswitched WDM networks," IEEE Journal on Selected Areas in Communications, Vol. 18, No. 10 , Oct. 2000 , pp. 1838-1851

[16] S. Baroni, P. Bayvel, "Wavelength requirements in arbitrarily connected wavelengthrouted optical networks," J. of Lightwave Technology, Vol.15, No.2, Feb. 1997, pp.242251

[17] M. Baldi, Y. Ofek, "End-to-end delay analysis of videoconferencing over packetswitched networks," IEEE/ACM Trans. on Networking, Vol. 8, No. 4, Aug. 2000, pp. 479492

[18] A. Myers, P. Bayvel, "Performance of the Just-Enough-Time (JET) Scheme for Optical Burst Switching", Proc. LCS 2001, London, Sep. 2001 